\title{
Anti-Inflammatory Mechanisms of Xuefu Zhuyu Decoction in The Treatment of Atherosclerosis Based on Network Pharmacology and Microarray Data Differences Analysis
}

Haoran Li

Shandong University of Traditional Chinese Medicine https://orcid.org/0000-0003-4346-5249

Hongyun Wu

Shandong University of Traditional Chinese Medicine Affiliated Hospital

Weiying Li

Weifang Maternal and Child Health Hospital

Jie Yang

Shandong University of Traditional Chinese Medicine Affiliated Hospital

Wei Peng ( $\square$ szypengwei@163.com )

Shandong University of Traditional Chinese Medicine Affiliated Hospital https://orcid.org/0000-00031384-9014

\section{Research}

Keywords: Xuefu Zhuyu, atherosclerosis, microarray data differences analysis, network pharmacology, molecular mechanism

Posted Date: February 12th, 2021

DOl: https://doi.org/10.21203/rs.3.rs-184004/v1

License: (c) (i) This work is licensed under a Creative Commons Attribution 4.0 International License. Read Full License 


\section{Abstract}

Background: Xuefu Zhuyu decoction is a traditional Chinese formula composed of eleven herbs, which has the effect of promoting blood circulation and removing blood stasis. In this study, the antiinflammatory mechanisms of Xuefu Zhuyu decoction in the treatment of atherosclerosis were studied utilizing network pharmacology, data mining, microarray data differences analysis and molecular docking.

Methods: Analyzing data from the TCMSP, the effective components and key targets of Xuefu Zhuyu decoction were screened out. Atherosclerosis-related genes were extracted from the disease databases and determined according to differences analysis. The component-target network was constructed and gene enrichment analysis, as well as topology analysis, were carried out. Finally, the affinity between the target and the effective components was verified by molecular docking.

Results: We screened 186 effective components of Xuefu Zhuyu decoction from TCMSP and obtained 126 targets. Through searching the disease databases and analyzing the results of differences analysis, two hundred and one atherosclerosis-related genes were obtained. After constructing the componenttarget network, it was found that Xuefu Zhuyu decoction played an anti-atherosclerotic role by acting on 21 targets. The results of enrichment analysis suggested that 21 key targets were mainly enriched in biological processes such as leukocyte adhesion and endothelial cell proliferation. The results of molecular docking showed that the key components of Xuefu Zhuyu decoction, have a good affinity with IL-6 and VEGFA.

Conclusions: Our bioinformatics analyses suggest that Xuefu Zhuyu decoction plays an antiatherosclerotic role by regulating biological processes such as leukocyte adhesion and endothelial cell proliferation. This study provides a theoretical basis for the further study of the indications of Xuefu Zhuyu decoction and the development of anti-atherosclerotic drugs.

\section{Introduction}

Atherosclerotic (AS) is the early pathological change of cardiovascular-cerebrovascular diseases, which has a high incidence in the middle-aged and elderly population. So far, the pathogenesis of AS has not been completely clear, there are many theories, involving a variety of risk factors. A large number of clinical and basic studies have suggested that hyperlipidemia, hypertension, hyperglycemia, hyperfibrinogen, hypercysteinemia, hyperuricemia, obesity, smoking and hypercoagulable state are risk factors for AS [1]. With the gradual entry of AS into people's field of vision, anti-atherosclerotic drugs are also developing. Chinese and western medicine have made a lot of research on the pathogenesis, prevention and treatment of AS. The mechanism of Western medicine in the treatment of AS is clear and has achieved significant effect, but the effect is often single, and the side effects are large. Chinese medicine, guided by the theory of Traditional Chinese Medicine, has unique advantages for complex 
diseases as AS. Recently, great progress has been made in the treatment of AS with traditional Chinese medicine.

In the long-term clinical practice of traditional Chinese medicine, it has been found that some natural herbs can reverse vascular endothelial injury. Due to the lack of research on the mechanism of action of traditional Chinese medicine, the wider application of traditional Chinese medicine in the world is often hindered. Studies have shown that XZD can exert its therapeutic effect on AS by reducing the level of endothelium-derived contractile substance, increasing the level of endothelium-derived vasodilator and reducing cell adhesion [2]. A systematic review and meta-analysis showed that XZD is more effective than antihypertensive drugs in improving total cholesterol, triglycerides, low-density lipoprotein, homocysteine and hemorheology [3]. XZD is a traditional Chinese formula to promote blood circulation created by Qingren Wang in Qing Dynasty. XZD is composed of eleven herbs: Persicae semen (Tao Ren), Rehmanniae radix praeparata (Di Huang), Cyathulae radix (Niu Xi), Platycodon grandiforus (Jie Geng), Chuanxiong rhizome (Chuan Xiong), Carthami flos (Hong Hua), Radix paeoniae rubra (Chi Shao), Aurantii fructus (Zhi Ke), Angelicae sinensis radix (Dang Gui), licorice (Gan Cao) and Radix bupleuri (Chai Hu). The composition of Xuefu Zhuyu decoction is complex and various, therefore network pharmacology is needed to understand the mechanism of AS treatment.

Eleven herbs in XZD contain a variety of active ingredients, each of which acts on a large number of targets so that XZD can play a therapeutic role through the regulation of a large network of targets. Network pharmacology can help us to understand the complex mechanism of XZD in the treatment of AS through the enrichment analysis of the target network. Through network pharmacology, we can intuitively find the key components in XZD, the targets at the center of the target network, the genes related to AS and the biological processes with the most significant enrichment results.

In this study, the component-target network of XZD and AS was constructed utilizing network pharmacology and microarray data differences analysis. Starting with the effective components of XZD, the relationship between its action targets and AS-related genes was studied, and the intersection of them was taken as the key targets for subsequent enrichment analysis. Finally, the affinity between the effective components of XZD and the key targets was verified by molecular docking.

\section{Materials And Methods}

\subsection{Preliminary screening of effective components}

The effective components of XZD were screened from the Traditional Chinese Medicine Systems Pharmacology Database and Analysis Platform (TCMSP) (https://tcmspw.com/tcmsp.php) including 499 herbs and 12144 compounds. The effective components of XZD were screened using the TCMSP with oral bioavailability $(O B) \geq 30 \%$ and drug-like properties $(D L) \geq 0.18$. The absorption of effective components of XZD from the gastrointestinal tract to the systemic circulation is a complete process, and $\mathrm{OB}$ is an index to evaluate the rate and degree of the process [4]. DL is an index of the possibility of a compound as a drug [5]. Due to the variety of traditional Chinese medicine ingredients, the absorption 
process of traditional Chinese medicine in the human body is very complex, the establishment of a unified standard for the evaluation of traditional Chinese medicine components is very important for screening the effective components of traditional Chinese medicine and the follow-up network pharmacological analysis.

\subsection{Acquisition of proteins targeted by XZD effective components}

The targets of the screened compounds were acquired from TCMSP and the DrugBank (https://go.drugbank.com) databases and standardized with gene symbols by the UniProt (https://www.uniprot.org/) database.

\subsection{Screening atherosclerosis-related target genes}

We retrieved four disease databases with the "atherosclerosis" to obtain the genes related to AS: Gene Expression Omnibus (GEO)( https://www.ncbi.nlm.nih.gov/geo/) $\bigotimes$ DisGeNET (https://www.disgenet.org), Therapeutic Target Database (TTD)( http://db.idrblab.net/ttd/) and Online Mendelian Inheritance in Man (OMIM)(https://omim.org/). In order to obtain the genes most significantly associated with AS, we have developed the following data mining strategies for four databases: in the OMIM and TTD databases, we searched within "scope of disease"; in the DisGeNET database, we screened genes with GDA scores greater than 0.1 ; in the GEO databases, we picked up a microarray expression profile dataset named GSE43292 from carotid intima for 32 hypertensive patients. We downloaded the original files of the gene chip, which were computed utilizing the LIMMA package in the R platform to find the differentially expressed genes (DEGs) according to the difference of gene expression between the control group and the plaque group [6]. The DEGs computed by the R platform were following the criteria of llog2 (fold change) $\mid \geq 1$ and $P<0.05$.

\subsection{Construction of component-target network, GO enrichment analysis and KEGG enrichment analysis of key genes}

We intersect the targets of XZD with the targets of AS-related genes and act as the key targets of XZD in the treatment of AS. Combined with the relationship between the effective components of XZD and the action targets in TCMSP above, the component-target network was constructed. After the key targets were analyzed by the online database STRING (https://string-db.org), they were imported into Cytoscape to draw the protein-protein interaction (PPI) network. The Degree is an index and basis used to describe the importance of targets in PPI and to draw network diagrams, which can help us to more intuitively find targets that play a key role in the biological process of XZD in the treatment of AS. We used the ClueGO app of Cytoscape for Gene Ontology (GO) enrichment analysis and Kyoto Encyclopedia of Genes and Genomes (KEGG) enrichment analysis (https://www.kegg.jp) to obtain the functional annotations of key genes and related pathways. GO enrichment analysis focused on biological processes.

\subsection{Molecular docking}


The effective components and key targets were docked by AutoDock Vina to verify their affinity [7]. Firstly, we downloaded the compounds structure files from the PubChem database and the structure files for the proteins from the PDB database. PyMOL was used to process proteins by removing waters and $\mathrm{SO}_{4}{ }^{2-}[8]$. AutoDock Tools was used to add polar hydrogens to the receptors and adjust the rotatable bond of the ligand. Discovery Studio was used to predict the site of the docking box because of the lacking of the original ligand of VEGFA, and the docking box size was set to $15 \times 15 \times 15$ (The spacing between each

grid point was $1 \AA$ ). Finally, the results were analyzed and plotted by Discovery Studio, and the calculated results of AutoDock Vina were derived as an index to evaluate the interaction activity.

\subsection{PPI network topology analysis and hub genes screening}

In order to study all possible roles of target genes in the pharmacological network, a topology network was constructed using the BisoGenet app in Cytoscape [9]. Subsequently, CytoNCA, a Cytoscape app for network centrality analysis, was used to screen hub genes in the network [10]. Firstly, CytoNCA was used to screen genes that were more than twice the median degree centrality (DC) value for subnetwork construction. Then, genes with the top $50 \%$ highest betweenness centrality (BC) values in the subnetwork were identified as hub genes and formed the core network.

\section{Results}

\subsection{Acquisition of effective components and their targets}

TCMSP was used to search for 11 herbs in Xuefu Zhuyu Decoction, and 186 effective components were screened out according to the standard of $\mathrm{OB} \geq 30$ and $\mathrm{DL} \geq 0.18$. An additional table file shows this in more detail [see Additional file 1]. The prediction targets of effective components were obtained by the PubChem database, and the standard gene symbol was given by UniProt. One hundred and twenty-six targets were obtained after excluding the targets without gene symbols. An additional table file shows this in more detail [see Additional file 2].

\subsection{Target genes associated with atherosclerosis}

A total of 82 AS-related genes were obtained by retrieving the TTD, OMIM and DisGeNET databases. Eighty AS-related genes were screened in DisGeNet (an additional table file shows this in more detail [see Additional file 3]), 0 in TTD and 2 in OMIM. Based on the analysis of the difference of gene expression in the series matrix (an additional table file shows this in more detail [see Additional file 4]), we obtained the DEGs (an additional table file shows this in more detail [see Additional file 5]) that meet the criteria llog2 (fold change) $\mid \geq 1$ and $P<0.05$ [11]. The analysis results revealed 148 DEGs including 86 up-regulated genes and 62 down-regulated genes, which were used to draw volcano maps and heat maps (Fig. 2). A total of 201 AS-related genes were obtained by summarizing the search results of OMIM, TTD and DisGeNET, and the differences analysis results of the GSE43292 dataset.

\subsection{Construction and PPI analysis of protein targets network}


We regard the intersection of effective component targets and disease-related targets as the targets of XZD in the treatment of AS. There were 21 targets in the intersection of the two networks, that is, XZD played a therapeutic role in AS by acting on 21 targets. The complex relationship between 21 targets and effective components was shown by the component-target network (Fig. 3a). After introducing the results of PPI analysis of 21 targets into Cytoscape, we found that the proteins encoded by IL6 and VEGFA were the most important in the PPI network (Fig. 3b).

\subsection{Biological function enrichment of gene targets in XZD treatment of AS}

GO enrichment analysis showed that 21 target genes were mainly enriched in leukocyte adhesion to the vascular endothelial cell, lipid storage, endothelial cell proliferation and other biological functions (Fig. 4a). And the target genes are mainly enriched in two aspects: acute inflammatory response and leukocyte adhesion to a vascular endothelial cell, accounting for $41.43 \%$ and $34.29 \%$ (Fig. 4b), respectively. Then we focused on the detailed analysis of the biological processes in $\mathrm{GO}$ enrichment analysis and found that the targets acted on the biological processes such as positive regulation of leukocyte adhesion to an arterial endothelial cell, negative regulation of endothelial cell development, interleukin-17 biosynthetic process, cellular response to vitamin E, et al (Fig. 4c). KEGG enrichment analysis showed that the gene enrichment on fluid shear stress and atherosclerosis signaling pathway was the most significant. Shear stress represents the friction force exerted by blood flow on the surface of vascular endothelial cells and plays an important role in the progress of AS [12]. Sustained laminar flow with high shear stress can resist AS. The disturbed flow with low shear stress can promote the oxidation and inflammation in the arterial wall, which participate in the process of AS. A total of eight genes were enriched in this signal pathway, including FOS, VCAM1, ICAM1, SELE, VEGFA, NOS3, GSTM1 and CAV1. FOS, VCAM1, ICAM1, SELE and VEGFA participate in the pro-atherogenesis, while NOS3, GSTM1 and CAV1 participate in the anti-atherogenesis (Fig. 5).

\subsection{Molecular docking results}

We selected the compounds with Degree $>5$ in the component-target network as the main components of XZD, selected IL6 and VEGFA in the PPI network as the key genes, and docked the proteins transcribed by the key genes with the main components respectively. We regard the affinity of interleukin- 6 and its original ligand TLA as the criterion to evaluate the degree of molecular binding. An affinity of less than -3 indicated a high interaction activity between ligand and receptor. The docking results (Table 1) showed that the affinity of the four main components to interleukin- 6 was less than that of the original ligand to interleukin-6, and the affinity of the four main components to vascular endothelial growth factor was less than -3 . Therefore, the four compounds had a good degree of binding to the target protein. The affinity of kaempferol to the target proteins was the lowest, and the docking results of kaempferol with the two target proteins were displayed by image (Fig. 6).

Table 1 Docking result of the compound on target. 


\begin{tabular}{|lcl|}
\hline Name & IL6 & VEGFA \\
\hline baicalein & -4.1 & -4.8 \\
\hline kaempferol & -4.1 & -5.5 \\
\hline luteolin & -3.5 & -5.5 \\
\hline quercetin & -3.3 & -5.4 \\
\hline original ligand & -3.0 & null \\
\hline
\end{tabular}

The four compounds were docked with two proteins respectively, and the affinity between them is at the intersection of row and column. The last row of the table is the affinity between the protein and its original ligand. The null value indicates that there is no original ligand of VEGFA.

\subsection{Network topology analysis results}

XZD had a therapeutic effect on AS by directly acting on 21 target proteins. To study the wider effects of XZD in the human body and the indirect targets of XZD, we carried out a topological analysis of 21 target proteins. After two times of screening, we obtained a network map of 180 hub genes in the topological analysis and found that the hub genes contained five XZD direct action targets: VCAM1, CAV1, PPARG, ESR1 and FOS (Fig. 7). An additional table file shows this in more detail [see Additional file 6].

\section{Discussion}

Xuefu Zhuyu decoction, a blood regulating agent, has the effect of promoting blood circulation and removing blood stasis, promoting qi and relieving pain. Modern pharmacological studies have confirmed that Xuefu Zhuyu decoction has the effects of improving hemorheology, protecting vascular endothelial function, inhibiting platelet aggregation, treating coronary heart disease, improving myocardial infarction, lowering blood pressure, regulating blood lipids, anti-AS and so on. Utilizing network pharmacology, we found that the anti-AS effect of XZD involves a complex component-target-pathway network.

The theories to explain the pathogenesis of AS include vascular smooth muscle cell clone theory [13], lipid infiltration theory [14], oxidative stress theory [15], platelet hyperfunction theory [16], thrombosis theory [17], immune function abnormality theory [18], shear stress theory [19], injury reaction theory [20], inflammation theory and so on [21]. Among these theories, the inflammatory mechanism of AS is a relatively new theory, and a lot of research work has been carried out. According to the results of GO enrichment analysis, KEGG enrichment analysis, PPI analysis and network topology analysis, this paper discussed the mechanism of XZD in the treatment of AS.

The development of AS can be divided into three stages: early stage, progressive stage and terminal stage. In the early stage of AS, vascular endothelial cells (VECs) are injured for various reasons, which stimulate VECs to express adhesion molecules and promote monocytes and lymphocytes to adhere to vascular endothelium and migrate to the intima [22]. A large number of low-density lipoproteins are 
modified into oxidized low-density lipoproteins (oxLDL) and accumulate in the vascular wall, accelerating the process of AS [23]. After entering the vascular wall, monocytes differentiate into macrophages, engulf oxLDL and transform into foam cells [24], which participate in the process of AS together with T cells, B cells and other lymphocytes. The progression stage of AS is actually a proliferative inflammation of the vascular wall. Under the influence of cytokines, vascular smooth muscle cells migrate from media to the intima and proliferate [13]. In the terminal stage of AS, activated natural killer T cells can cause VECs death or apoptosis [25], coupled with the dissolution of collagen fibers in plaque extracellular matrix by matrix metalloproteinases [26], resulting in plaque rupture, bleeding and thrombosis. From the enrichment analysis of key targets, XZD has an influence on the early, progressive and terminal stages of AS.

\subsection{Biomarkers}

Atherosclerosis involves many biomarkers, such as cytokines, adhesion molecules, C-reactive protein, matrix metalloproteinases and so on. Interleukin-6 (IL-6) is a cytokine involves in the expression and regulation of the immunity system and serves to acute and chronic inflammatory responses[27]. Related clinical studies have shown that IL- 6 is related to the incidence of cardiovascular events and mortality. The level of IL-6 in patients with AS was significantly higher than that in healthy people, and the level of IL-6 is positively correlated with the severity of the disease [28]. The level of IL- 6 increased significantly in patients with plaque rupture [29]. Quercetin exerts an anti-inflammatory effect by inhibiting the neutrophil secretion of IL- 6 and strongly inhibits mast cell secretion of IL- 6 by inhibiting p38 signaling pathway and PKC phosphorylation [30,31]. Luteolin down-regulates the expression of IL-6 in monocytes by inhibiting MAPKs and NF-kB signaling pathways and reduces the secretion of IL-6 in microglia by inhibiting JNK phosphorylation and activating AP-1 [32,33].

When the inflammatory response system is activated, macrophages gather in the vascular endothelium and release a large amount of IL- 6 and tumor necrosis factor-a, inducing the liver to synthesize a large amount of C-reactive protein (CRP) [34,35]. CRP is not present in healthy vascular walls, but it can be detected in the early stages of AS, binds to low-density lipoprotein, exists in atherosclerotic plaques and gradually accumulates $[36,37]$. CRP promotes the recruitment of monocytes to the vascular wall by increasing the secretion of endothelial cell adhesion molecules and promotes plaque instability by inducing the expression of matrix metalloproteinase (MMPs) [38-40]. CRP up-regulates the expression of vascular endothelial growth factor (VEGF) by activating MMP-2, which significantly promotes the proliferation of vascular endothelial cells [41]. CRP also contributes to thrombosis [42]. In addition, CRP is considered to be a predictor of cardiovascular events, and CRP levels can independently predict the risk of cardiovascular death in the general population [43]. A meta-analysis of available randomized controlled trials showed a significant reduction of circulating CRP levels with quercetin [44]. Quercetin inhibits the recruitment of monocytes by reducing the level of CRP in plasma, and inhibits the proliferation of vascular endothelial cells and stabilizes plaques by regulating the expression of matrix metalloproteinases induced by CRP. Its therapeutic effect runs through the early, progressive and terminal stages of AS. 
Adhesion molecules play an important role in the process of AS. [45]. Adhesion molecules are proteins that mediate cell-cell and cell-extracellular matrix contact and adhesion. They can be divided into selectin family, integrin family and immunoglobulin superfamily (IgSF) which can be regulated by XZD [46]. Selectin is the first step in leukocyte adhesion in inflammatory, and E-selectin (SELE) supports leukocyte adhesion to vascular endothelial cells [45]. Vascular cell adhesion molecule-1 (VCAM-1) and intercellular adhesion molecule-1 (ICAM-1), which can promote the early adhesion of monocytes to vascular endothelial cells, are members of the immunoglobulin superfamily and ligands of integrins $[47,48]$. The expression of VCAM- 1 and ICAM- 1 is induced by cytokines such as tumor necrosis factor- $a$ (TNF- $a$ ) and interleukin-1 (IL-1) [49,50]. The components of XZD, including quercetin, luteolin and kaempferol, interfere with the adhesion of monocytes to vascular endothelial cells in the early stage of AS by regulating the expression of SELE, VCAM1 and ICAM1. Quercetin and kaempferol have direct effects on the three adhesion molecules.

Vascular endothelial growth factor A (VEGFA) plays an important role in vascular development and neovascularization in physiological and pathological processes. VEGFA and its receptor VEGFR are involved in the progression of AS. In the early stage of AS, VEGFA secreted by macrophages acts on both VEGFR-1 and VEGFR-2 receptors [51]. VEGFR-1 induces monocyte migration [52], thus enabling macrophages differentiated by monocytes to gather in the plaque, while VEGFR-2 accelerates the process of AS by stimulating vascular growth [53]. Many studies have shown that intimal neovascularization is related to the development and stability of plaques [54-57]. Quercetin, beta-carotene, luteolin, baicalein and ellagic acid can regulate the expression of VEGFA, thus affecting monocyte recruitment and vascular endothelial cell proliferation, delaying the early stage of AS.

\subsection{Signaling pathways}

In the results of KEGG enrichment analysis, we found that three signaling pathways have an important impact on the process of AS, namely Toll-like receptor (TLR) signaling pathway, Nuclear factor-kappa B (NF-kB) signaling pathway and Janus kinase-signal transducer and activator of transcription (JAK-STAT) signaling pathway. In vascular smooth muscle cells, exogenous lipopolysaccharides activate the TLR4 signaling pathway, which activates NF-kB and promotes the release of cytokines such as monocyte chemoattractant protein-1 (MCP-1) and interleukin-6 (IL6) [58,59]. TLR4 can also induce local lipid accumulation by down-regulating the expression of ATP-binding cassette sub-family $\mathrm{G}$ member 1 (ABCG1) [60]. Although TLR4 is not directly regulated by the effective components of XZD, it is of great significance to the progress of AS and is an ideal target to interfere with the progression of AS. NF-kB signaling pathway is associated with inflammation and apoptosis in the progression of AS [61], which can be activated by inflammatory cytokines, adhesion molecules (Such as VCAM-1 and ICAM-1) and chemokines [62]. Down-regulation of NF-kB leads to a decrease in foam cell formation [63], while activation of the TLR4/NF-kB signaling pathway promotes plaque growth and reduces plaque stability [64]. Mice with high NF-kB expression increase the risk of atherosclerotic plaque formation in the aorta [65]. Although the enrichment of XZD targets in the NF-kB signaling pathway was not significant, the NF$\mathrm{kB}$ signaling pathway plays an important role in the progression of AS and is an important way to 
interfere with AS. JAK-STAT signaling pathway is an important signaling pathway regulating the early and terminal stages of AS [66]. JAK-STAT signaling pathway is activated by cytokines and participates in immune regulation in the progression of AS [67]. STAT6 activated by IL-4 promotes the differentiation of Thelper (Th) cells into Th2. STAT4 activated by IL-12 promotes the differentiation of Th cells into Th4. Th2 has the activity of anti-atherogenesis [68], while Th1 has the activity of pro-atherogenesis, which mediates the activation of macrophages and promotes the expansion of atherosclerotic plaque [69]. JAKSTAT signaling pathway plays a role in the bidirectional regulation of AS.

\subsection{Estrogen receptor in the network topology analysis}

In the network topology analysis, ESR1 was in the most critical position in the network with a Degree of 764. We found that ESR1 can regulate some biological processes in the early stage of AS. Estrogen receptor (ESR) inhibits the promoter of IL6 by disrupting the transactivation of NF-kB [70,71], thereby inhibiting the synthesis of $\mathrm{C}$-reactive protein and the proliferation of vascular endothelial cells [72]. ER46, the splice isomer of estrogen receptor, can activate endothelial nitric oxide synthase (eNOS) and promotes NO production [73]. The biological processes such as oxidative modification of low-density lipoproteins, a proliferation of vascular endothelial cells and infiltration of macrophages in AS are inhibited by NO [74]. ESR1 not only occupies the most critical position in topology analysis but also plays an important role in the component-target network. Eighty-five of the 101 effective components of XZD have a direct effect on ESR1. According to the results of network topology analysis, some of the nodes with higher Degree values were not the direct targets of the effective components of XZD. They have protein-protein interaction with the targets of effective components and thus participate in the regulation of AS by XZD. NTRK1 participates in the regulation of angiogenesis and the positive regulation of NF-kB transcription factor activity [75]. FN1 participates in the process of cell adhesion and cell-matrix adhesion $[76,77]$. However, the correlation between more targets in topological analysis and the pathogenesis of AS needs to be further studied.

The effective components and targets of XZD came from TCMSP and DrugBank databases, and the related genes of AS came from DisGeNET and GEO databases. The research in this paper was based on the known information in the database, and unknown compounds, targets and disease-related genes were not discussed in this paper. Therefore, this paper has certain timeliness.

\section{Conclusion}

The results suggest that the effective components of XZD acting on 21 atherosclerosis-related target genes can play an anti-atherosclerotic effect by regulating biological processes such as leukocyte adhesion and endothelial cell proliferation. In this paper, the anti-atherosclerotic effect of XZD was comprehensively analyzed utilizing data mining, difference analysis and molecular docking, and a large number of data were obtained, which provided evidence for the further application of XZD and a reference for the further study of the pathogenesis of atherosclerosis. 


\section{Abbreviations}

XZD, Xuefu Zhuyu decoction;

AS, atherosclerotic;

$\mathrm{OB}$, oral bioavailability;

DL, drug-like properties;

GEO, Gene Expression Omnibus;

OMIM, Mendelian Inheritance in Man;

TTD, Therapeutic Target Database;

DEGs, differentially expressed genes;

PPI, protein-protein interaction;

GO, Gene Ontology;

KEGG, Kyoto Encyclopedia of Genes and Genomes;

DC, degree centrality;

BC, betweenness centrality;

CC, closeness centrality;

VECs, vascular endothelial cells;

oxLDL, oxidized low-density lipoproteins;

IL-6, Interleukin-6;

CRP, C-reactive protein;

MMPs, matrix metalloproteinase;

VEGF, vascular endothelial growth factor;

ICAM-1, Intercellular adhesion molecule-1;

VCAM-1, vascular cell adhesion molecule-1;

NF-kB, Nuclear factor-kappa B; 
TLR, Toll-like receptor;

JAK-STAT, Janus kinase-signal transducer and activator of transcription;

ESR, Estrogen receptor.

\section{Declarations}

\section{Acknowledgements}

Professor Wei Peng is the provider of the funds, and her support for our report is very crucial.

\section{Authors' contributions}

(I) Conception and design: Wei Peng; (II) Administrative support: Wei Peng; (III) Provision of study materials or patients: Weiying Li; (IV) Collection and assembly of data: Haoran Li, Hongyun Wu, Jie Yang; (V) Data analysis and interpretation: Haoran Li; (VI) Manuscript writing: All authors; (VII) Final approval of manuscript: All authors.

\section{Funding}

This article is supported by the special scientific research project of the business construction of the National TCM Clinical Research Base of the State Administration of Traditional Chinese Medicine (NO. JDZX2015105).

\section{Availability of data and materials}

The datasets supporting the conclusions of this article are included within the article and its additional files.

\section{Ethics approval and consent to participate}

Not applicable.

\section{Consent for publication}

Not applicable.

\section{Competing interests}

The authors declare that they have no conflict of interests.

\section{Author emails}

Haoran Li: dc812050413@163.com; Hongyun Wu: 19286045@qq.com; Weiying Li:

Iwy13563664800@163.com; Jie Yang: 13583121098@163.com; Wei Peng: szypengwei@163.com. 


\section{References}

1. Ji X, Leng X-Y, Dong Y, Ma Y-H, Xu W, Cao X-P, et al. Modifiable risk factors for carotid atherosclerosis: a meta-analysis and systematic review. Ann Transl Med. 2019;7:632.

2. Wang BX, Dong XM, Guo AM, Zhang J. Effects of xuefu zhuyu decoction on functions of vascular endothelium in patients with unstable angina pectoris. Zhong Xi Yi Jie He Xue Bao. China; 2006;4:256-9.

3. Wang P, Xiong X, Li S. Efficacy and Safety of a Traditional Chinese Herbal Formula Xuefu Zhuyu Decoction for Hypertension: A Systematic Review and Meta-Analysis. Medicine (Baltimore). 2015;94:1-18.

4. Xu X, Zhang W, Huang C, Li Y, Yu H, Wang Y, et al. A novel chemometric method for the prediction of human oral bioavailability. Int J Mol Sci. 2012;13:6964-82.

5. Tao W, Xu X, Wang X, Li B, Wang Y, Li Y, et al. Network pharmacology-based prediction of the active ingredients and potential targets of Chinese herbal Radix Curcumae formula for application to cardiovascular disease. J Ethnopharmacol. Ireland; 2013;145:1-10.

6. Ritchie ME, Phipson B, Wu D, Hu Y, Law CW, Shi W, et al. limma powers differential expression analyses for RNA-sequencing and microarray studies. Nucleic Acids Res. 2015;43:e47.

7. Trott O, Olson AJ. AutoDock Vina: improving the speed and accuracy of docking with a new scoring function, efficient optimization, and multithreading. J Comput Chem. 2010;31:455-61.

8. Seeliger D, de Groot BL. Ligand docking and binding site analysis with PyMOL and Autodock/Vina. J Comput Aided Mol Des. 2010;24:417-22.

9. Martin A, Ochagavia ME, Rabasa LC, Miranda J, Fernandez-de-Cossio J, Bringas R. BisoGenet: a new tool for gene network building, visualization and analysis. BMC Bioinformatics. 2010;11:91.

10. Tang Y, Li M, Wang J, Pan Y, Wu F-X. CytoNCA: a cytoscape plugin for centrality analysis and evaluation of protein interaction networks. Biosystems. Ireland; 2015;127:67-72.

11. Ayari $\mathrm{H}$, Bricca $\mathrm{G}$. Identification of two genes potentially associated in iron-heme homeostasis in human carotid plaque using microarray analysis. J Biosci. India; 2013;38:311-5.

12. Malek AM, Alper SL, Izumo S. Hemodynamic shear stress and its role in atherosclerosis. JAMA. United States; 1999;282:2035-42.

13. Chistiakov DA, Orekhov AN, Bobryshev Y V. Vascular smooth muscle cell in atherosclerosis. Acta Physiol (Oxf). England; 2015;214:33-50.

14. Usman A, Ribatti D, Sadat U, Gillard JH. From Lipid Retention to Immune-Mediate Inflammation and Associated Angiogenesis in the Pathogenesis of Atherosclerosis. J Atheroscler Thromb. Japan; 2015;22:739-49.

15. Kattoor AJ, Pothineni NVK, Palagiri D, Mehta JL. Oxidative Stress in Atherosclerosis. Curr Atheroscler Rep. United States; 2017;19:42.

16. Custodio-Chablé SJ, Lezama RA, Reyes-Maldonado E. Platelet activation as a trigger factor for inflammation and atherosclerosis. Cir Cir. Mexico; 2020;88:233-43. 
17. Holvoet P, Collen D. Thrombosis and atherosclerosis. Curr Opin Lipidol. England; 1997;8:320-8.

18. Hansson GK, Libby P. The immune response in atherosclerosis: a double-edged sword. Nat Rev Immunol. England; 2006;6:508-19.

19. Souilhol C, Serbanovic-Canic J, Fragiadaki M, Chico TJ, Ridger V, Roddie H, et al. Endothelial responses to shear stress in atherosclerosis: a novel role for developmental genes. Nat Rev Cardiol. England; 2020;17:52-63.

20. Mannarino E, Pirro M. Endothelial injury and repair: a novel theory for atherosclerosis. Angiology. United States; 2008;59:69S-72S.

21. Geovanini GR, Libby P. Atherosclerosis and inflammation: overview and updates. Clin Sci (Lond). England; 2018;132:1243-52.

22. Zhu Y, Xian X, Wang Z, Bi Y, Chen Q, Han X, et al. Research Progress on the Relationship between Atherosclerosis and Inflammation. Biomolecules. 2018;8.

23. Maiolino G, Rossitto G, Caielli P, Bisogni V, Rossi GP, Calò LA. The role of oxidized low-density lipoproteins in atherosclerosis: the myths and the facts. Mediators Inflamm. 2013;2013:714653.

24. Chistiakov DA, Melnichenko AA, Myasoedova VA, Grechko A V, Orekhov AN. Mechanisms of foam cell formation in atherosclerosis. J Mol Med (Berl). Germany; 2017;95:1153-65.

25. Getz GS, Reardon CA. Natural killer T cells in atherosclerosis. Nat Rev Cardiol. England; 2017;14:304-14.

26. Johnson JL. Metalloproteinases in atherosclerosis. Eur J Pharmacol. Netherlands; 2017;816:93-106.

27. Tanaka T, Narazaki M, Kishimoto T. Interleukin (IL-6) Immunotherapy. Cold Spring Harb Perspect Biol. 2018;10.

28. Aker S, Bantis C, Reis P, Kuhr N, Schwandt C, Grabensee B, et al. Influence of interleukin-6 G-174C gene polymorphism on coronary artery disease, cardiovascular complications and mortality in dialysis patients. Nephrol Dial Transplant Off Publ Eur Dial Transpl Assoc - Eur Ren Assoc. England; 2009;24:2847-51.

29. Held C, White HD, Stewart RAH, Budaj A, Cannon CP, Hochman JS, et al. Inflammatory Biomarkers Interleukin-6 and C-Reactive Protein and Outcomes in Stable Coronary Heart Disease: Experiences From the STABILITY (Stabilization of Atherosclerotic Plaque by Initiation of Darapladib Therapy) Trial. J Am Heart Assoc. 2017;6.

30. Liu J, Li X, Yue Y, Li J, He T, He Y. The inhibitory effect of quercetin on IL-6 production by LPSstimulated neutrophils. Cell Mol Immunol. China; 2005;2:455-60.

31. Kandere-Grzybowska K, Kempuraj D, Cao J, Cetrulo CL, Theoharides TC. Regulation of IL-1-induced selective IL- 6 release from human mast cells and inhibition by quercetin. $\mathrm{Br} \mathrm{J}$ Pharmacol. 2006;148:208-15.

32. Jang S, Kelley KW, Johnson RW. Luteolin reduces IL-6 production in microglia by inhibiting JNK phosphorylation and activation of AP-1. Proc Natl Acad Sci U S A. 2008;105:7534-9. 
33. Lee YS, Kim MS, Lee DH, Kwon TH, Song H-H, Oh S-R, et al. Luteolin 8-C- $\beta$-fucopyranoside downregulates IL-6 expression by inhibiting MAPKs and the NF-KB signaling pathway in human monocytic cells. Pharmacol Rep. Switzerland; 2015;67:581-7.

34. Sproston NR, Ashworth JJ. Role of C-Reactive Protein at Sites of Inflammation and Infection. Front Immunol. 2018;9:754.

35. Boras E, Slevin M, Alexander MY, Aljohi A, Gilmore W, Ashworth J, et al. Monomeric C-reactive protein and Notch-3 co-operatively increase angiogenesis through PI3K signalling pathway. Cytokine. England; 2014;69:165-79.

36. Badimon L, Peña E, Arderiu G, Padró T, Slevin M, Vilahur G, et al. C-Reactive Protein in Atherothrombosis and Angiogenesis. Front Immunol. 2018;9:430.

37. Blaha MJ, Rivera JJ, Budoff MJ, Blankstein R, Agatston A, O'Leary DH, et al. Association between obesity, high-sensitivity C-reactive protein $\geq 2 \mathrm{mg} / \mathrm{L}$, and subclinical atherosclerosis: implications of JUPITER from the Multi-Ethnic Study of Atherosclerosis. Arterioscler Thromb Vasc Biol. 2011;31:1430-8.

38. Cimmino G, Ragni M, Cirillo P, Petrillo G, Loffredo F, Chiariello M, et al. C-reactive protein induces expression of matrix metalloproteinase-9: a possible link between inflammation and plaque rupture. Int J Cardiol. Netherlands; 2013;168:981-6.

39. Hattori Y, Matsumura M, Kasai K. Vascular smooth muscle cell activation by C-reactive protein. Cardiovasc Res. England; 2003;58:186-95.

40. Kawanami D, Maemura K, Takeda N, Harada T, Nojiri T, Saito T, et al. C-reactive protein induces VCAM-1 gene expression through NF-kappaB activation in vascular endothelial cells. Atherosclerosis. Ireland; 2006;185:39-46.

41. Chen J, Gu Z, Wu M, Yang Y, Zhang J, Ou J, et al. C-reactive protein can upregulate VEGF expression to promote ADSC-induced angiogenesis by activating HIF-1a via CD64/PI3k/Akt and MAPK/ERK signaling pathways. Stem Cell Res Ther. 2016;7:114.

42. Cermak J, Key NS, Bach RR, Balla J, Jacob HS, Vercellotti GM. C-reactive protein induces human peripheral blood monocytes to synthesize tissue factor. Blood. United States; 1993;82:513-20.

43. Kaptoge S, Di Angelantonio E, Pennells L, Wood AM, White IR, Gao P, et al. C-reactive protein, fibrinogen, and cardiovascular disease prediction. N Engl J Med. 2012;367:1310-20.

44. Mohammadi-Sartang M, Mazloom Z, Sherafatmanesh S, Ghorbani M, Firoozi D. Effects of supplementation with quercetin on plasma C-reactive protein concentrations: a systematic review and meta-analysis of randomized controlled trials. Eur J Clin Nutr. England; 2017;71:1033-9.

45. Price DT, Loscalzo J. Cellular adhesion molecules and atherogenesis. Am J Med. United States; 1999;107:85-97.

46. McMurray RW. Adhesion molecules in autoimmune disease. Semin Arthritis Rheum. United States; 1996;25:215-33.

47. Albelda SM, Oliver PD, Romer LH, Buck CA. EndoCAM: a novel endothelial cell-cell adhesion molecule. J Cell Biol. 1990;110:1227-37. 
48. Newman PJ, Berndt MC, Gorski J, White GC 2nd, Lyman S, Paddock C, et al. PECAM-1 (CD31) cloning and relation to adhesion molecules of the immunoglobulin gene superfamily. Science. United States; 1990;247:1219-22.

49. Pober JS, Gimbrone MAJ, Lapierre LA, Mendrick DL, Fiers W, Rothlein R, et al. Overlapping patterns of activation of human endothelial cells by interleukin 1, tumor necrosis factor, and immune interferon. J Immunol. United States; 1986;137:1893-6.

50. Dustin ML, Rothlein R, Bhan AK, Dinarello CA, Springer TA. Induction by IL 1 and interferon-gamma: tissue distribution, biochemistry, and function of a natural adherence molecule (ICAM-1). J Immunol. United States; 1986;137:245-54.

51. Inoue $M$, Itoh $H$, Ueda $M$, Naruko $T$, Kojima A, Komatsu R, et al. Vascular endothelial growth factor (VEGF) expression in human coronary atherosclerotic lesions: possible pathophysiological significance of VEGF in progression of atherosclerosis. Circulation. United States; 1998;98:2108-16.

52. Shibuya M. VEGF-VEGFR System as a Target for Suppressing Inflammation and other Diseases. Endocr Metab Immune Disord Drug Targets. United Arab Emirates; 2015;15:135-44.

53. Celletti FL, Waugh JM, Amabile PG, Brendolan A, Hilfiker PR, Dake MD. Vascular endothelial growth factor enhances atherosclerotic plaque progression. Nat Med. United States; 2001;7:425-9.

54. Barger AC, Beeuwkes R 3rd, Lainey LL, Silverman KJ. Hypothesis: vasa vasorum and neovascularization of human coronary arteries. A possible role in the pathophysiology of atherosclerosis. N Engl J Med. United States; 1984;310:175-7.

55. Moulton KS, Heller E, Konerding MA, Flynn E, Palinski W, Folkman J. Angiogenesis inhibitors endostatin or TNP-470 reduce intimal neovascularization and plaque growth in apolipoprotein Edeficient mice. Circulation. United States; 1999;99:1726-32.

56. Moulton KS, Vakili K, Zurakowski D, Soliman M, Butterfield C, Sylvin E, et al. Inhibition of plaque neovascularization reduces macrophage accumulation and progression of advanced atherosclerosis. Proc Natl Acad Sci U S A. 2003;100:4736-41.

57. Kolodgie FD, Gold HK, Burke AP, Fowler DR, Kruth HS, Weber DK, et al. Intraplaque hemorrhage and progression of coronary atheroma. N Engl J Med. United States; 2003;349:2316-25.

58. Fitzgerald KA, Kagan JC. Toll-like Receptors and the Control of Immunity. Cell. United States; 2020;180:1044-66.

59. Hollestelle SCG, De Vries MR, Van Keulen JK, Schoneveld AH, Vink A, Strijder CF, et al. Toll-like receptor 4 is involved in outward arterial remodeling. Circulation. United States; 2004;109:393-8.

60. O'Shea JJ, Pesu M, Borie DC, Changelian PS. A new modality for immunosuppression: targeting the JAK/STAT pathway. Nat Rev Drug Discov. England; 2004;3:555-64.

61. Berliner JA, Navab M, Fogelman AM, Frank JS, Demer LL, Edwards PA, et al. Atherosclerosis: basic mechanisms. Oxidation, inflammation, and genetics. Circulation. United States; 1995;91:2488-96.

62. Gwon W-G, Joung E-J, Kwon M-S, Lim S-J, Utsuki T, Kim H-R. Sargachromenol protects against vascular inflammation by preventing TNF-a-induced monocyte adhesion to primary endothelial cells via inhibition of NF-KB activation. Int Immunopharmacol. Netherlands; 2017;42:81-9. 
63. Kanters E, Pasparakis M, Gijbels MJJ, Vergouwe MN, Partouns-Hendriks I, Fijneman RJA, et al. Inhibition of NF-kappaB activation in macrophages increases atherosclerosis in LDL receptordeficient mice. J Clin Invest. 2003;112:1176-85.

64. Zeng X, Guo R, Dong M, Zheng J, Lin H, Lu H. Contribution of TLR4 signaling in intermittent hypoxiamediated atherosclerosis progression. J TransI Med. 2018;16:106.

65. Hajra L, Evans Al, Chen M, Hyduk SJ, Collins T, Cybulsky MI. The NF-kappa B signal transduction pathway in aortic endothelial cells is primed for activation in regions predisposed to atherosclerotic lesion formation. Proc Natl Acad Sci U S A. 2000;97:9052-7.

66. Grote K, Luchtefeld M, Schieffer B. JANUS under stress-role of JAK/STAT signaling pathway in vascular diseases. Vascul Pharmacol. United States; 2005;43:357-63.

67. Recinos A 3rd, LeJeune WS, Sun H, Lee CY, Tieu BC, Lu M, et al. Angiotensin II induces IL-6 expression and the Jak-STAT3 pathway in aortic adventitia of LDL receptor-deficient mice. Atherosclerosis. 2007;194:125-33.

68. Tabas I, Lichtman AH. Monocyte-Macrophages and T Cells in Atherosclerosis. Immunity. 2017;47:621-34.

69. Ridker PM, Rifai N, Pfeffer MA, Sacks F, Braunwald E. Long-term effects of pravastatin on plasma concentration of C-reactive protein. The Cholesterol and Recurrent Events (CARE) Investigators. Circulation. United States; 1999;100:230-5.

70. Liu H, Liu K, Bodenner DL. Estrogen receptor inhibits interleukin-6 gene expression by disruption of nuclear factor kappaB transactivation. Cytokine. England; 2005;31:251-7.

71. Stein B, Yang MX. Repression of the interleukin- 6 promoter by estrogen receptor is mediated by NFkappa B and C/EBP beta. Mol Cell Biol. 1995;15:4971-9.

72. Hartman J, Frishman WH. Inflammation and atherosclerosis: a review of the role of interleukin-6 in the development of atherosclerosis and the potential for targeted drug therapy. Cardiol Rev. United States; 2014;22:147-51.

73. Kim KH, Toomre D, Bender JR. Splice isoform estrogen receptors as integral transmembrane proteins. Mol Biol Cell. 2011;22:4415-23.

74. Förstermann U, Xia N, Li H. Roles of Vascular Oxidative Stress and Nitric Oxide in the Pathogenesis of Atherosclerosis. Circ Res. United States; 2017;120:713-35.

75. Tacconelli A, Farina AR, Cappabianca L, Desantis G, Tessitore A, Vetuschi A, et al. TrkA alternative splicing: a regulated tumor-promoting switch in human neuroblastoma. Cancer Cell. United States; 2004;6:347-60.

76. Gaudet P, Livstone MS, Lewis SE, Thomas PD. Phylogenetic-based propagation of functional annotations within the Gene Ontology consortium. Brief Bioinform. 2011;12:449-62.

77. Main AL, Harvey TS, Baron M, Boyd J, Campbell ID. The three-dimensional structure of the tenth type III module of fibronectin: an insight into RGD-mediated interactions. Cell. United States; 1992;71:671-8. 


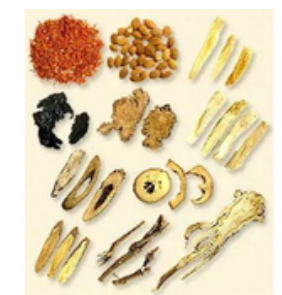

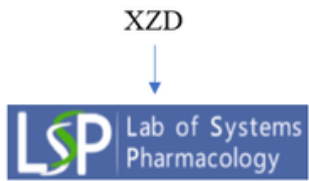

Preliminary Screening of Effective Components

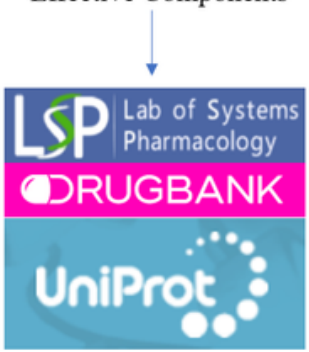

Acquisition of standardized targets

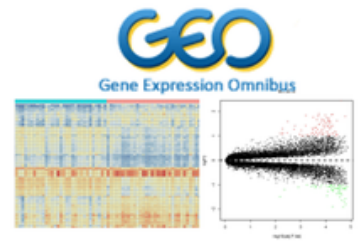

Microarray data differences analysis based on GEO database
Therapeutic Target Database

OMIM $^{8}$

Online Mendelian Inheritance in Man

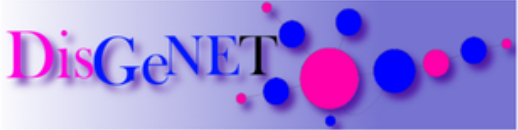

Screening atherosclerosis genes

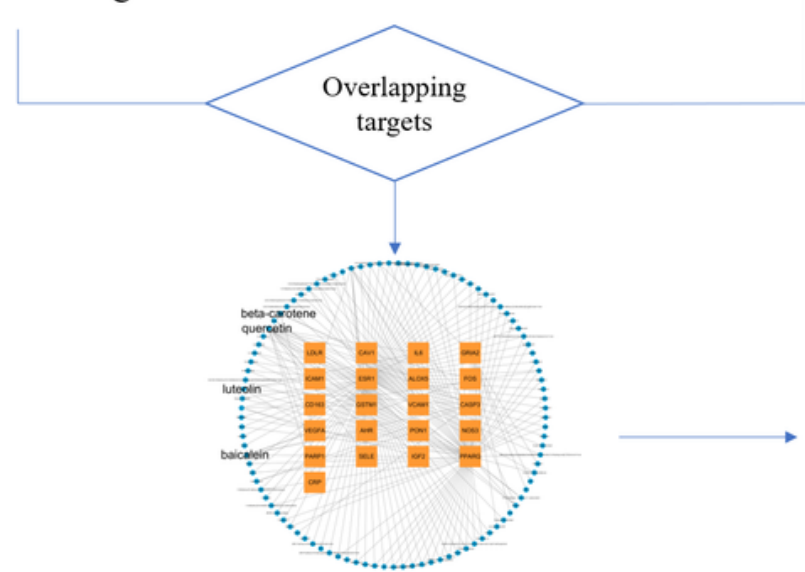

Network analysis

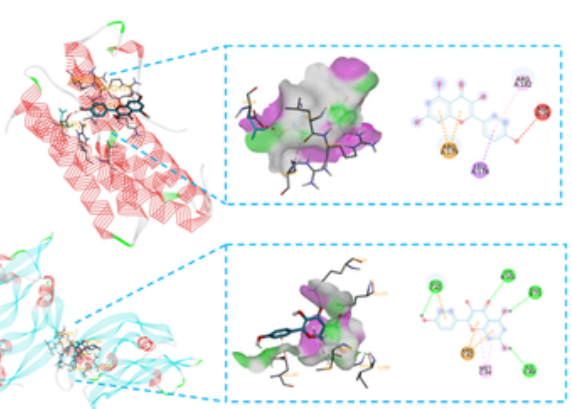

The effective components and key targets were docked by AutoDockVina to verify their affinity.

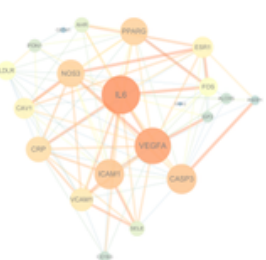

PPI network

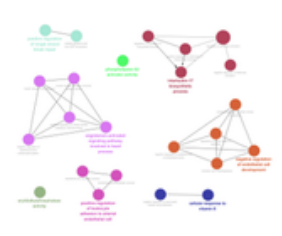

GO enrichment analysis

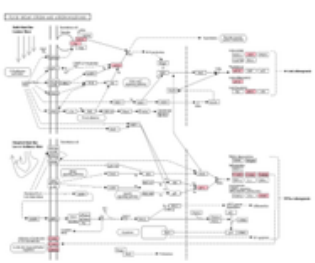

GO enrichment analysis

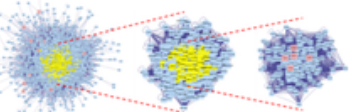

Topology analysis

\section{Figure 1}

Overall workflow of this study 

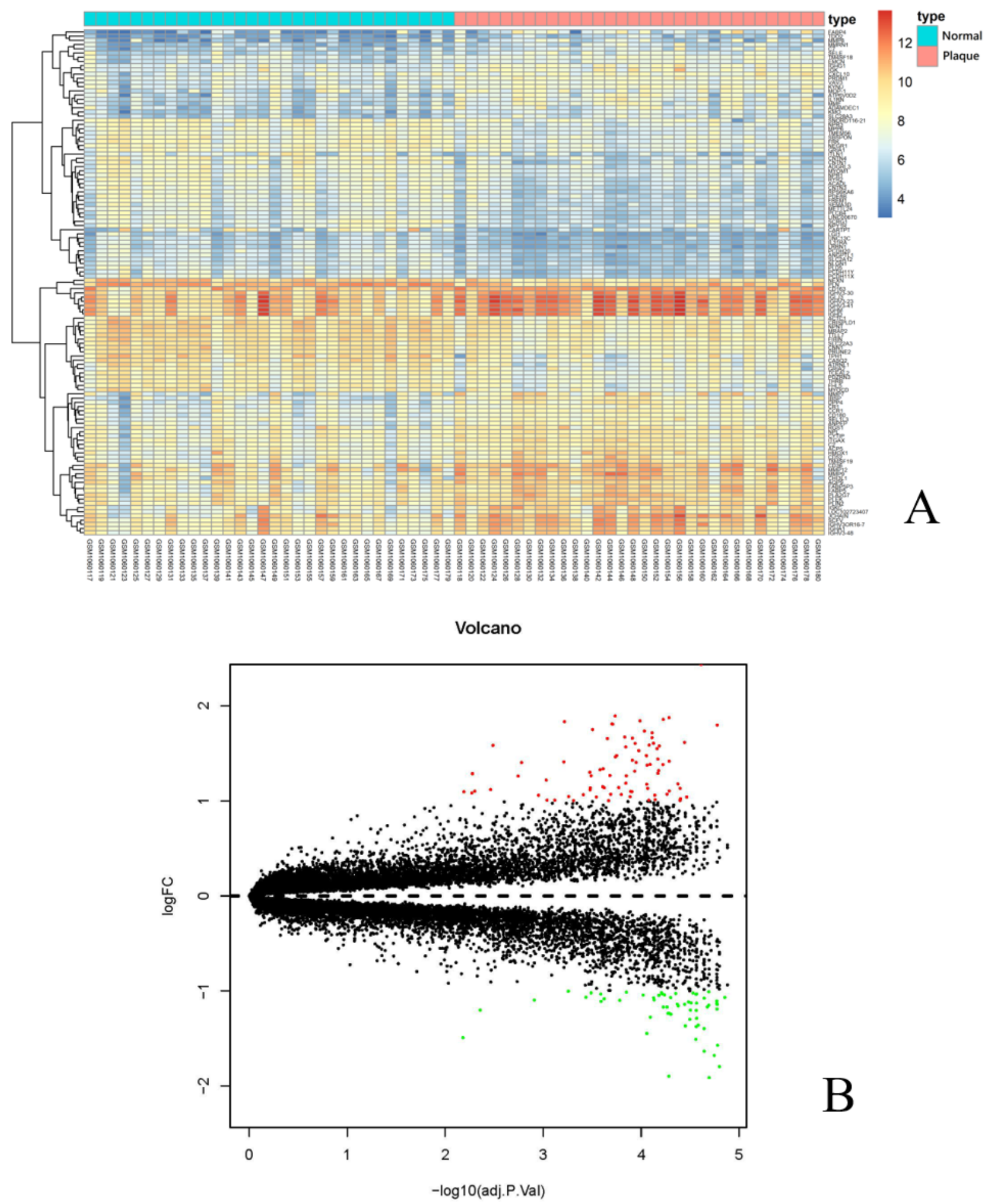

Figure 2

The results of microarray data differences analysis. a Heat map of differentially expressed genes (DEGs) between the control group and the plaque group. The redder the color is, the higher the gene expression is, and the bluer the color is, the lower the gene expression is. b DEGs volcano map between the control group and the plaque group. Red represents up-regulated genes and the green represents down-regulated genes. 


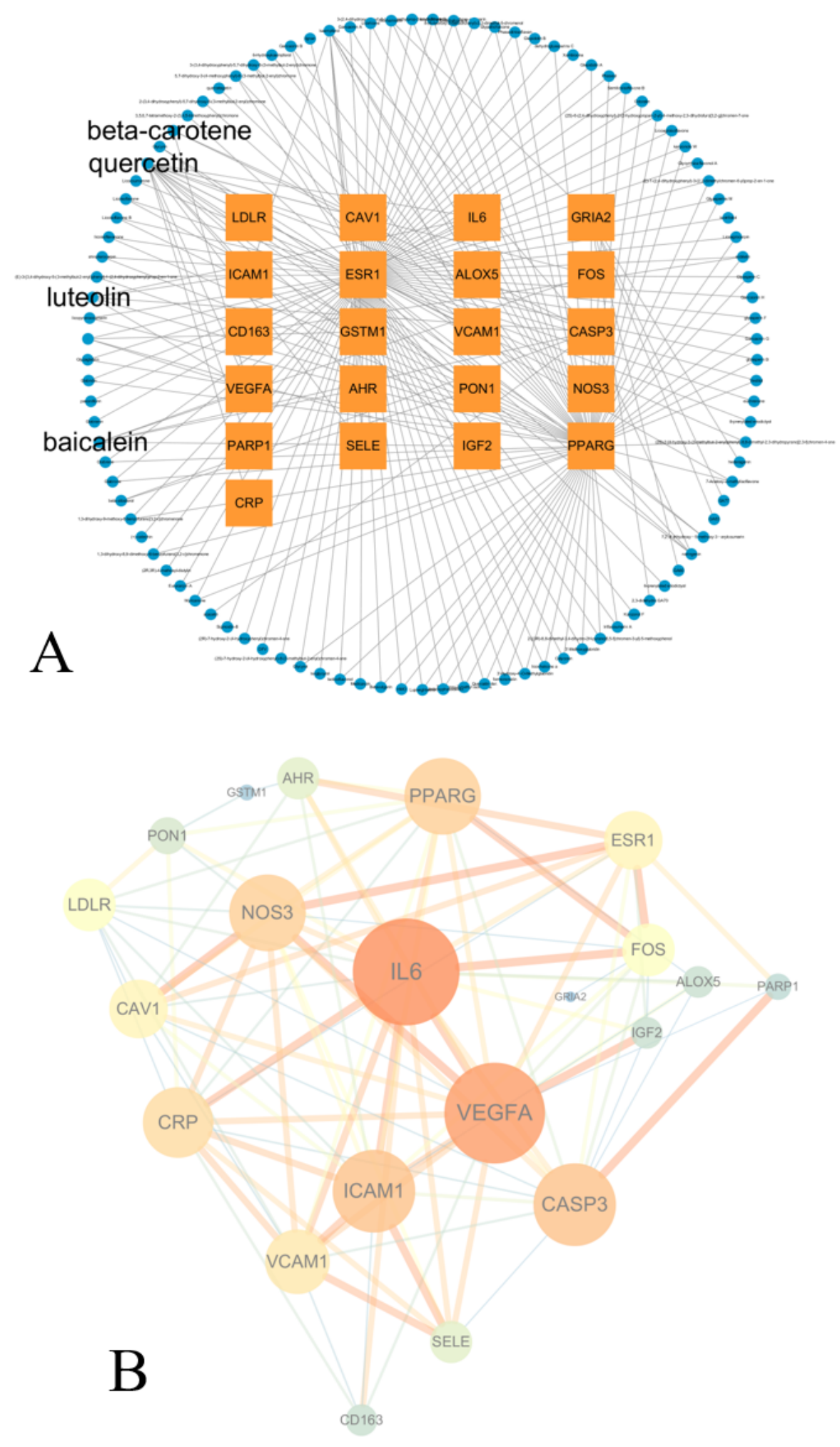

\section{Figure 3}

Network pharmacology analysis results. A Component-target network. The blue circle represents the effective components, and the orange square represents the target. The edge represents the existence of molecular interactions. B Protein-protein interaction (PPI) network of XZD. The size and brightness of nodes are related to Degree. 


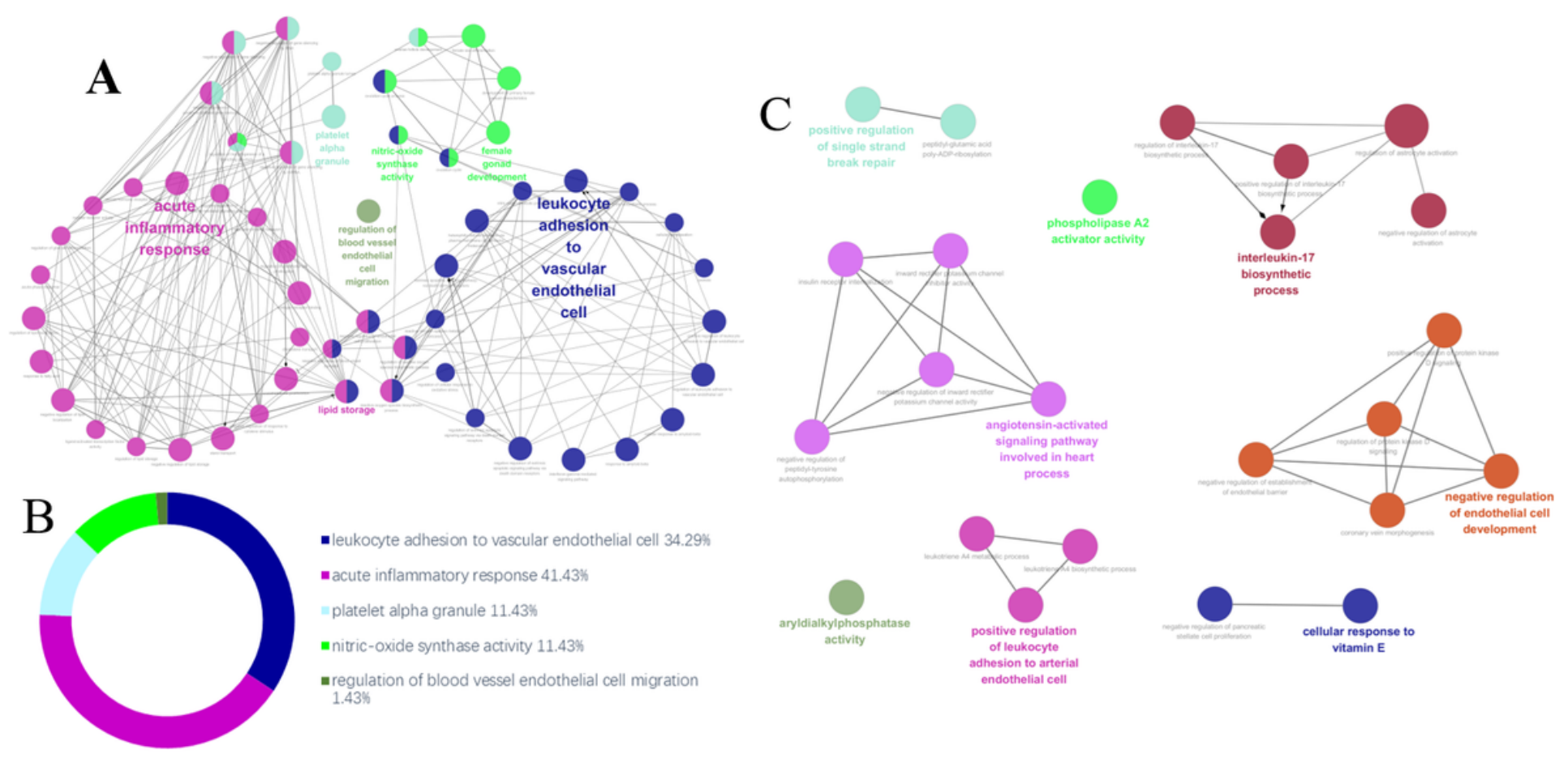

Figure 4

The GO enrichment analysis. Different colors represent different types of biological functions. a The network of GO enrichment analysis. b GO enrichment analysis pie chart. c The biological process of GO enrichment analysis. 
FLUID SHEAR STRESS AND ATHEROSCLEROSIS

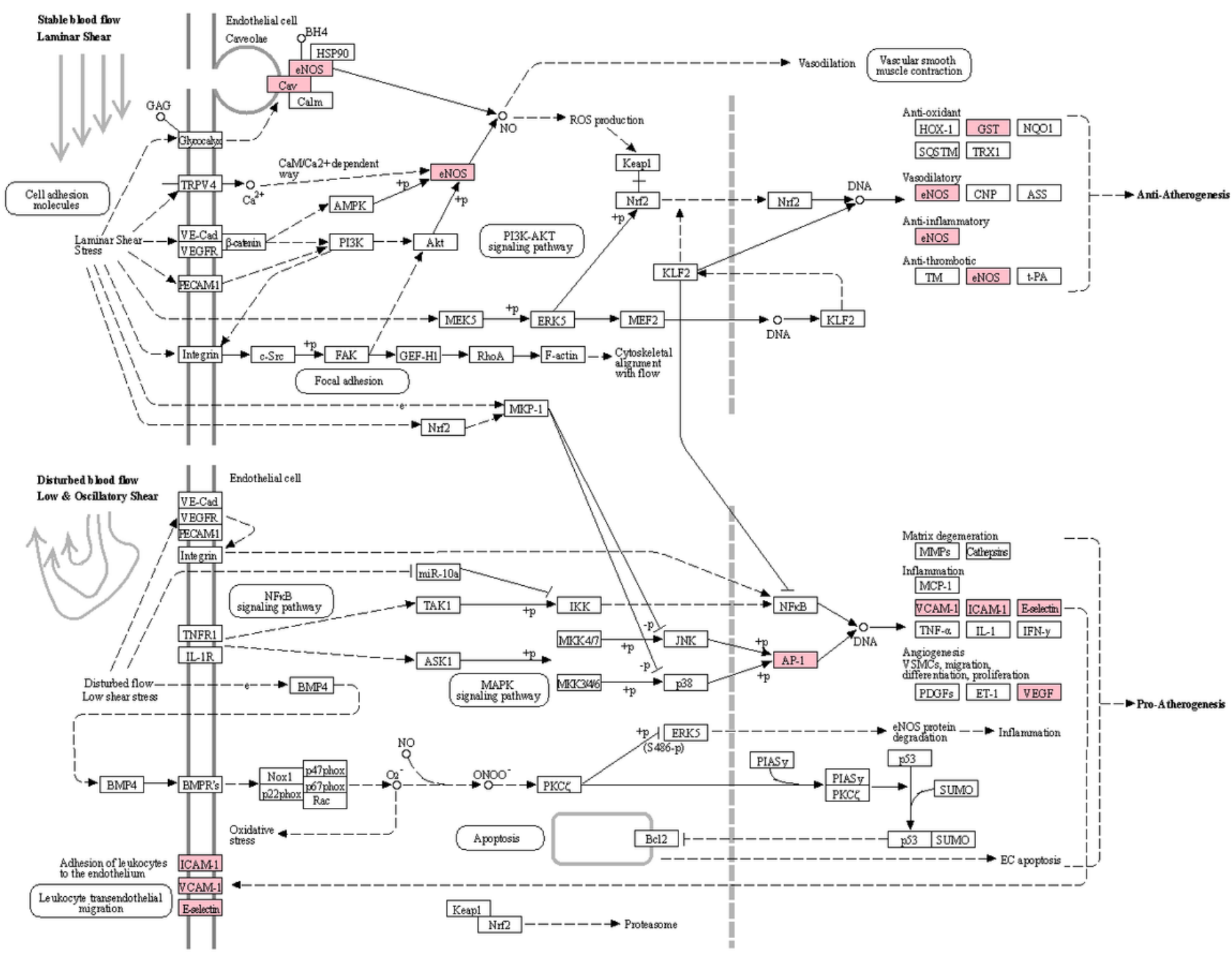

Figure 5

The KEGG pathways enrichment analysis. The red square is the biomarkers represented by the targets of XZD. 

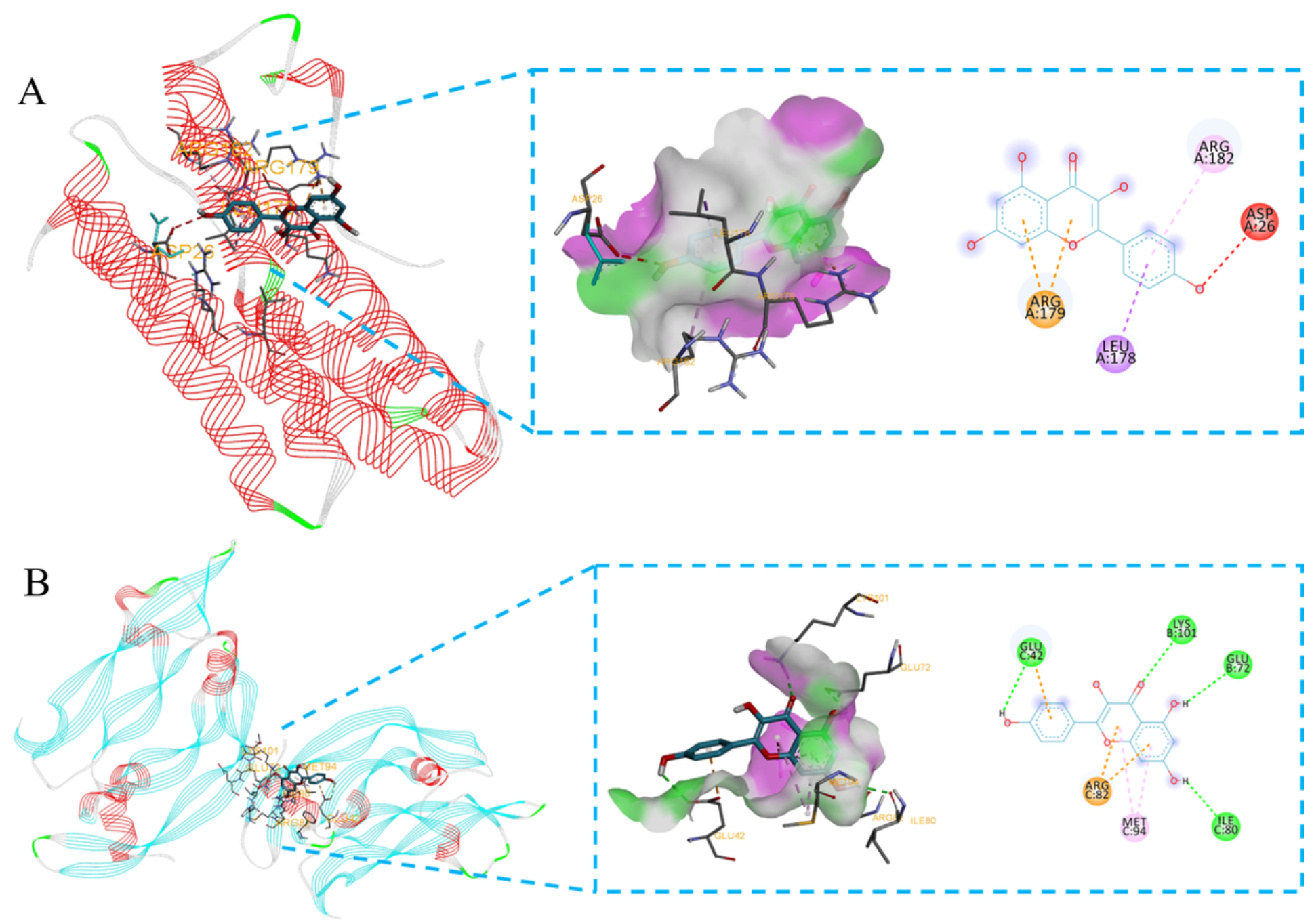

Figure 6

Molecular docking results. a Molecular docking results of kaempferol and IL6. b Molecular docking results of kaempferol and vascular endothelial growth factor (VEGFA).

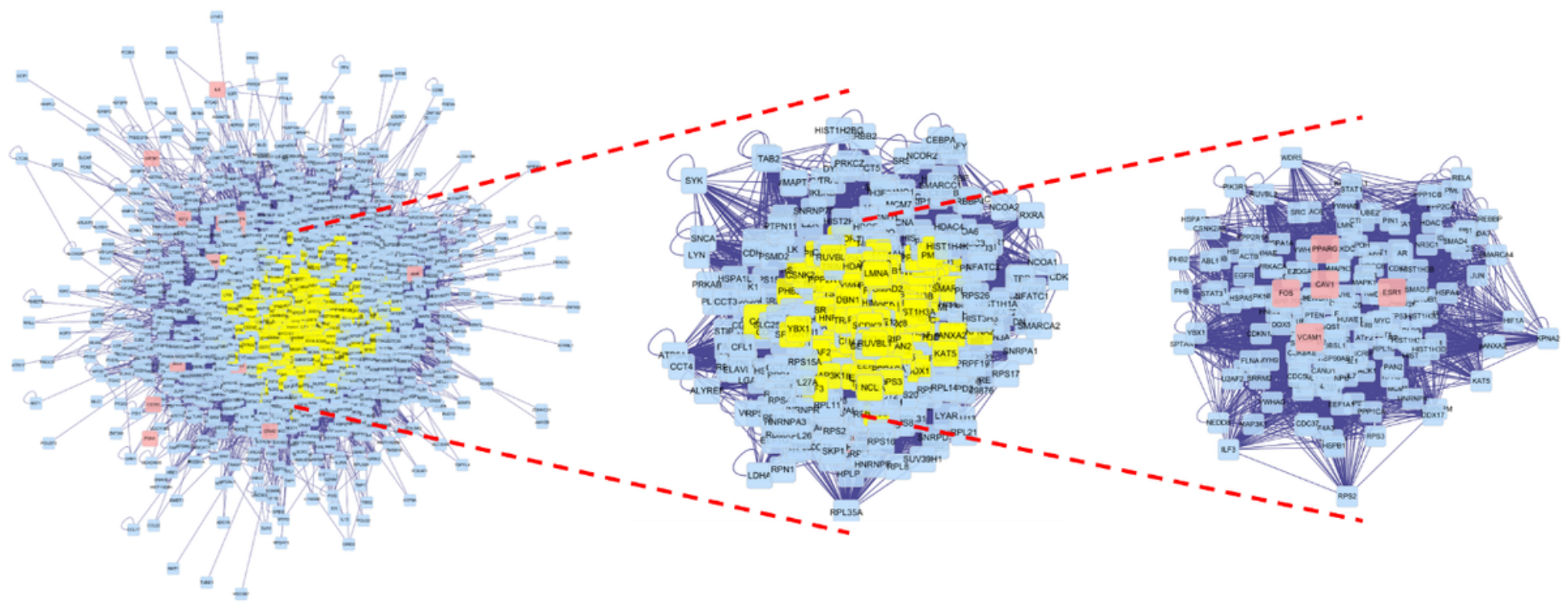


Figure 7

Network topology analysis. The hub gene network was obtained by twice screening in topology analysis.

\section{Supplementary Files}

This is a list of supplementary files associated with this preprint. Click to download.

- Additionalfile1.docx

- Additionalfile2.docx

- Additionalfile3.xlsx

- Additionalfile4.xlsx

- Additionalfile5.xlsx

- Additionalfile6.xIsx 